Supplement of Biogeosciences Discuss., 11, 18145-18188, 2014

http://www.biogeosciences-discuss.net/11/18145/2014/

doi:10.5194/bgd-11-18145-2014-supplement

(C) Author(s) 2014. CC Attribution 3.0 License.

(c) (i)

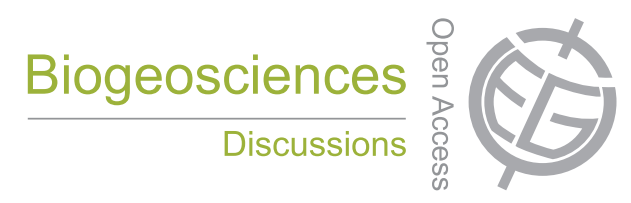

Supplement of

\title{
The trophic and metabolic pathways of foraminifera in the Arabian Sea: evidence from cellular stable isotopes
}

R. M. Jeffreys et al.

Correspondence to: R. M. Jeffreys (rachel.jeffreys@liv.ac.uk) 
Table S1: Station data for deployments from both margins referred to in the Methods and Figure 1. Exact sampling positions, depths, gear type used and the sample fate.

\begin{tabular}{|c|c|c|c|c|c|c|}
\hline Margin & Discovery \# & Latitude (N) & Longitude (E) & Depth (m) & Gear & Sample Type \\
\hline Oman & $55704 \# 2$ & $23^{\circ} 29.57$ & $59^{\circ} 14.06$ & 3101 & CTD & DO profile \\
\hline Oman & $55708 \# 1$ & $23^{\circ} 41.47$ & $58^{\circ} 42.32$ & 1435 & CTD & DO profile \\
\hline Oman & $55726 \# 3$ & $23^{\circ} 19.99$ & $59^{\circ} 06.81$ & 1935 & CTD & DO profile \\
\hline Oman & $55730 \# 1$ & $22^{\circ} 46.40$ & $59^{\circ} 57.30$ & 2000 & CTD & DO profile \\
\hline Oman & $55786 \# 1$ & $22^{\circ} 58.01$ & $60^{\circ} 08.21$ & 3145 & CTD & DO profile \\
\hline Oman & $55712 \# 1$ & $23^{\circ} 23.07$ & $58^{\circ} 59.03$ & 334 & Megacore & Bulk sediment organics \\
\hline Oman & $55717 \# 1$ & $23^{\circ} 21.18$ & $59^{\circ} 03.19$ & 1341 & Megacore & Bulk sediment organics \\
\hline Oman & $55718 \# 3$ & $23^{\circ} 21.67$ & $58^{\circ} 59.30$ & 800 & Megacore & Bulk sediment organics \\
\hline Oman & $55725 \# 2$ & $23^{\circ} 20.31$ & $59^{\circ} 09.85$ & 2010 & Megacore & Bulk sediment organics \\
\hline Oman & $55726 \# 1$ & $23^{\circ} 19.87$ & $59^{\circ} 07.34$ & 2075 & Megacore & Bulk sediment organics \\
\hline Oman & $55730 \# 2$ & $22^{\circ} 46.40$ & $59^{\circ} 57.52$ & 1980 & Megacore & Bulk sediment organics \\
\hline Oman & $55753 \# 1$ & $23^{\circ} 23.47$ & $59^{\circ} 03.51$ & 1093 & Megacore & Bulk sediment organics \\
\hline Oman & $55731 \# 1$ & $22^{\circ} 41.51$ & $59^{\circ} 56.01$ & 1414 & Megacore & Foraminiferal isotopes \\
\hline Oman & $55754 \# 1$ & $23^{\circ} 22.98$ & $59^{\circ} 00.00$ & 504 & Megacore & Foraminiferal isotopes \\
\hline Oman & $55763 \# 1$ & $23^{\circ} 23.02$ & $58^{\circ} 59.15$ & 363 & Megacore & Foraminiferal isotopes \\
\hline Oman & $55765 \# 1$ & $23^{\circ} 22.76$ & $59^{\circ} 02.05$ & 789 & Megacore & Foraminiferal isotopes \\
\hline Oman & $55766 \# 1$ & $23^{\circ} 23.52$ & $59^{\circ} 03.55$ & 1103 & Megacore & Foraminiferal isotopes \\
\hline Oman & $55767 \# 1$ & $23^{\circ} 22.10$ & $59^{\circ} 05.60$ & 1390 & Megacore & Foraminiferal isotopes \\
\hline Oman & $55788 \# 1$ & $23^{\circ} 01.00$ & $60^{\circ} 02.98$ & 3185 & Megacore & Foraminiferal isotopes \\
\hline Oman & $55789 \# 3$ & $22^{\circ} 22.49$ & $60^{\circ} 06.35$ & 2030 & Megacore & Foraminiferal isotopes \\
\hline Pakistan & $56001 \# 1$ & $22^{\circ} 52.34$ & $65^{\circ} 59.58$ & 1849 & CTD & DO profile/POM organics \\
\hline Pakistan & $56011 \# 2$ & $22^{\circ} 59.99$ & $66^{\circ} 24.43$ & 1197 & CTD & DO profile/POM organics \\
\hline Pakistan & $56020 \# 1$ & $22^{\circ} 55.82$ & $66^{\circ} 36.34$ & 930 & CTD & DO profile/POM organics \\
\hline Pakistan & $56028 \# 1$ & $23^{\circ} 12.36$ & $66^{\circ} 33.98$ & 307 & CTD & DO profile/POM organics \\
\hline Pakistan & $56036 \# 3$ & $23^{\circ} 16.43$ & $66^{\circ} 42.76$ & 136 & CTD & DO profile/POM organics \\
\hline
\end{tabular}




\begin{tabular}{|c|c|c|c|c|c|c|}
\hline Pakistan & $56065 \# 1$ & $22^{\circ} 52.46$ & $66^{\circ} 00.02$ & 1847 & CTD & DO profile/POM organics \\
\hline Pakistan & $56044 \# 3$ & $22^{\circ} 59.92$ & $66^{\circ} 24.06$ & 1211 & CTD & DO profile/POM organics \\
\hline Pakistan & $56031 \# 3$ & $23^{\circ} 16.52$ & $66^{\circ} 42.40$ & 134 & Megacore & Bulk sediment organics \\
\hline Pakistan & $56033 \# 4$ & $23^{\circ} 16.76$ & $66^{\circ} 42.64$ & 136 & Megacore & Bulk sediment organics \\
\hline Pakistan & $56016 \# 3$ & $23^{\circ} 16.92$ & $66^{\circ} 42.74$ & 134 & Megacore & Bulk sediment organics \\
\hline Pakistan & $56101 \# 10$ & $23^{\circ} 16.77$ & $66^{\circ} 42.71$ & 131 & Megacore & Bulk sediment organics \\
\hline Pakistan & $56025 \# 04$ & $23^{\circ} 12.48$ & $66^{\circ} 34.02$ & 310 & Megacore & Bulk sediment organics \\
\hline Pakistan & $56025 \# 05$ & $23^{\circ} 12.54$ & $66^{\circ} 34.03$ & 307 & Megacore & Bulk sediment organics \\
\hline Pakistan & $56025 \# 06$ & $23^{\circ} 12.21$ & $66^{\circ} 33.88$ & 314 & Megacore & Bulk sediment organics \\
\hline Pakistan & $56107 \# 01$ & $23^{\circ} 12.48$ & $66^{\circ} 34.00$ & 300.5 & Megacore & Bulk sediment organics \\
\hline Pakistan & $56015 \# 02$ & $22^{\circ} 55.90$ & $66^{\circ} 36.12$ & 958 & Megacore & Bulk sediment organics \\
\hline Pakistan & $56012 \# 03$ & $22^{\circ} 55.84$ & $66^{\circ} 36.10$ & 956 & Megacore & Bulk sediment organics \\
\hline Pakistan & $56015 \# 03$ & $22^{\circ} 55.88$ & $66^{\circ} 36.09$ & 960 & Megacore & Bulk sediment organics \\
\hline Pakistan & $56116 \# 03$ & $22^{\circ} 53.52$ & $66^{\circ} 36.66$ & 942 & Megacore & Bulk sediment organics \\
\hline Pakistan & $56007 \# 02$ & $22^{\circ} 59.76$ & $66^{\circ} 24.24$ & 1215 & Megacore & Bulk sediment organics \\
\hline Pakistan & $56007 \# 04$ & $22^{\circ} 59.78$ & $66^{\circ} 24.49$ & 1203 & Megacore & Bulk sediment organics \\
\hline Pakistan & $56007 \# 03$ & $22^{\circ} 59.91$ & $66^{\circ} 24.41$ & 1205 & Megacore & Bulk sediment organics \\
\hline Pakistan & $56141 \# 02$ & $23^{\circ} 00.00$ & $66^{\circ} 24.42$ & 1198 & Megacore & Bulk sediment organics \\
\hline Pakistan & $56005 \# 02$ & $22^{\circ} 52.52$ & $66^{\circ} 00.11$ & 1860 & Megacore & Bulk sediment organics \\
\hline Pakistan & $56005 \# 04$ & $22^{\circ} 52.26$ & $66^{\circ} 59.80$ & 1864 & Megacore & Bulk sediment organics \\
\hline Pakistan & $56001 \# 04$ & $22^{\circ} 52.55$ & $66^{\circ} 59.93$ & 1860 & Megacore & Bulk sediment organics \\
\hline Pakistan & $56137 \# 13$ & $22^{\circ} 52.39$ & $66^{\circ} 59.94$ & 1853 & Megacore & Bulk sediment organics \\
\hline Pakistan & $56101 \# 10$ & $23^{\circ} 16.77$ & $66^{\circ} 42.71$ & 132 & Megacore & Foraminiferal isotopes \\
\hline Pakistan & $56101 \# 04$ & $23^{\circ} 16.79$ & $66^{\circ} 42.70$ & 133 & Megacore & Foraminiferal isotopes \\
\hline Pakistan & $56105 \# 05$ & $23^{\circ} 12.49$ & $66^{\circ} 34.01$ & 285.4 & Megacore & Foraminiferal isotopes \\
\hline Pakistan & $56116 \# 04$ & $22^{\circ} 53.53$ & $66^{\circ} 36.64$ & 943 & Megacore & Foraminiferal isotopes \\
\hline Pakistan & $56116 \# 06$ & $22^{\circ} 53.52$ & $66^{\circ} 36.64$ & 943 & Megacore & Foraminiferal isotopes \\
\hline Pakistan & $56137 \# 01$ & $22^{\circ} 52.39$ & $66^{\circ} 00.03$ & 1853 & Megacore & Foraminiferal isotopes \\
\hline Pakistan & $56137 \# 02$ & $22^{\circ} 52.41$ & $66^{\circ} 00.04$ & 1853 & Megacore & Foraminiferal isotopes \\
\hline Pakistan & 56139\#01 & $22^{\circ} 59.99$ & $66^{\circ} 24.41$ & 1192 & Megacore & Foraminiferal isotopes \\
\hline Pakistan & 56139\#02 & $22^{\circ} 59.98$ & $66^{\circ} 24.42$ & 1193 & Megacore & Foraminiferal isotopes \\
\hline
\end{tabular}


Table S2: $\delta^{13} \mathrm{C}$ and $\delta^{15} \mathrm{~N}$ composition of foraminifera given at species level, from both the Oman and Pakistan margins. Depth (m), dissolved oxygen ( $\left.\mu \mathrm{m}\right)$, OMZ zone (see manuscript text) and foraminiferal type are all given. The Tubothalamea marked $\mathrm{i}$ and ii and have agglutinated and calcareous walls, respectively.

\begin{tabular}{|c|c|c|c|c|c|c|c|c|}
\hline Margin & Depth (m) & $\begin{array}{c}\text { Oxygen } \\
(\mu \mathrm{m})\end{array}$ & Zone & Family & Species & $8^{13} \mathrm{C}$ & $8^{15} \mathrm{~N}$ & Type \\
\hline Oman & 363 & 14.73 & UTZ & Vaginulinidae & Amphicoryna sublineata & n.d. & 3.2 & Calcareous \\
\hline Oman & 504 & 5.1 & CORE & Vaginulinidae & Lenticulina sp. & n.d. & 7.0 & Calcareous \\
\hline Oman & 789 & 6.25 & CORE & Buliminidae & Globobulimina turgida & n.d. & 6.3 & Calcareous \\
\hline Oman & 789 & 6.25 & CORE & Eggerellidae & Karreriella sp. & -20.0 & 5.3 & Textulariid \\
\hline Oman & 789 & 6.25 & CORE & Eggerellidae & Karreriella sp. & -22.3 & 5.5 & Textulariid \\
\hline Oman & 1103 & 16.51 & LB & Foraminifera & unidentified & n.d. & 8.8 & \\
\hline Oman & 1103 & 16.51 & LB & Nonionidae & Nonionella sp. & -21.6 & 7.2 & Calcareous \\
\hline Oman & 1390 & 27.2 & LB & Hormosinidae & Reophax spp. & n.d. & 0.7 & Textulariid \\
\hline Oman & 1390 & 27.2 & LB & Ammodiscidae & Ammodiscus sp. & n.d. & 2.1 & i'Tubothalamea \\
\hline Oman & 1390 & 27.2 & LB & Haplophragmoididae & Veleroninoides scitulus & n.d. & 3.5 & Textulariid \\
\hline Oman & 1390 & 27.2 & LB & Planulinidae & Hyalina baltica & n.d. & 4.7 & Calcareous \\
\hline Oman & 1390 & 27.2 & LB & Parrelloididae & Cibicidoides sp. & n.d. & 5.3 & Calcareous \\
\hline Oman & 1390 & 27.2 & LB & Uvigerinidae & Uvigerina hollicki & -19.0 & 7.8 & Calcareous \\
\hline Oman & 1414 & 29.9 & LB & Chilostomellidae & Chilostomella oolina & n.d. & 1.8 & Calcareous \\
\hline Oman & 1414 & 29.9 & LB & Parrelloididae & Cibicidoides sp. & n.d. & 5.7 & Calcareous \\
\hline Oman & 1414 & 29.9 & LB & Planulinidae & Hyalina baltica & -11.5 & 6.8 & Calcareous \\
\hline Oman & 1414 & 29.9 & LB & Uvigerinidae & Uvigerina hollicki & n.d. & 8.7 & Calcareous \\
\hline Oman & $\begin{array}{c}1390+ \\
1414 \\
\end{array}$ & $27-30$ & LB & Buliminidae & Globobulimina sp. & n.d. & 3.3 & Calcareous \\
\hline Oman & $\begin{array}{c}1390+ \\
1414\end{array}$ & $27-30$ & LB & Hauerinidae & Pyrgo depressa & n.d. & 4.2 & Calcareous \\
\hline
\end{tabular}




\begin{tabular}{|c|c|c|c|c|c|c|c|c|}
\hline Oman & 2030 & 90.58 & BELOW & $\begin{array}{l}\text { Allogromida incertae } \\
\text { sedis }\end{array}$ & unidentified & -20.0 & 7.1 & Monothalamid \\
\hline Oman & 2030 & 90.58 & BELOW & Buliminidae & Globobulimina sp. & n.d. & 7.1 & Calcareous \\
\hline Oman & 2030 & 90.58 & BELOW & Foraminifera & unidentified & n.d. & 10.8 & \\
\hline Oman & 2030 & 90.58 & BELOW & Hauerinidae & Quinqueloculina sp. & n.d. & 6.6 & ${ }^{\mathrm{ii}}$ Tubothalamea \\
\hline Oman & 2030 & 90.58 & BELOW & Hormosinidae & Reophax dentaliniformis & -19.4 & 6.2 & Textulariid \\
\hline Oman & 2030 & 90.58 & BELOW & Hormosinidae & Reophax dentaliniformis & n.d. & 7.8 & Textulariid \\
\hline Oman & 2030 & 90.58 & BELOW & Psammosphaeridae & Psammosphaera fusca with Ammolagena clavata & -20.1 & 8.3 & Monothalamid \\
\hline Oman & 2030 & 90.58 & BELOW & Psammosphaeridae & Psammosphaera fusca with Ammolagena clavata & -20.2 & 5.4 & Monothalamid \\
\hline Oman & 2030 & 90.58 & BELOW & Uvigerinidae & Uvigerina hollicki & -20.1 & 4.1 & Calcareous \\
\hline Oman & 2030 & 90.58 & BELOW & Valvulinidae & Clavulina communis var. sp. & -20.3 & 9.3 & Textulariid \\
\hline Oman & 3185 & 166 & BELOW & Komokiidae & Lana sp. & -21.3 & 8.5 & Monothalamid \\
\hline Oman & 3185 & 166 & BELOW & Buliminidae & Globobulimina sp. & -20.7 & 3.1 & Calcareous \\
\hline Oman & 3185 & 166 & BELOW & Haplophragmoididae & Veleroninoides scitulus & -20.1 & 9.0 & Textulariid \\
\hline Oman & 3185 & 166 & BELOW & Rhabdamminidae & Rhizammina sp. & -21.2 & 10.0 & Monothalamid \\
\hline Oman & 3185 & 166 & BELOW & Rhabdamminidae & Rhizammina sp. & -20.7 & -2.6 & Monothalamid \\
\hline Oman & 3185 & 166 & BELOW & Uvigerinidae & Uvigerina hollicki & n.d. & -7.8 & Calcareous \\
\hline Pakistan & 140 & 4.9 & SHZ & Astrorhizidae & Pelosina sp. & -21.1 & 9.7 & Monothalamid \\
\hline Pakistan & 140 & 4.9 & SHZ & Buliminidae & Globobulimina cf. G. pyrula & -24.5 & 20.2 & Calcareous \\
\hline Pakistan & 140 & 4.9 & SHZ & $\begin{array}{l}\text { Allogromida incertae } \\
\text { sedis }\end{array}$ & unidentified & -20.5 & 11.5 & Monothalamid \\
\hline Pakistan & 140 & 4.9 & SHZ & Rhabdamminidae & Bathysiphon sp. & -23.3 & -3.9 & Monothalamid \\
\hline Pakistan & 140 & 4.9 & $\mathrm{SHZ}$ & & Bolivina ex. gr. dilatata \& Cassidulina laevigata & -20.5 & n.d. & Calcareous \\
\hline Pakistan & 140 & 4.9 & SHZ & Bagginidae & Cancris auriculus & -23.2 & 16.6 & Calcareous \\
\hline Pakistan & 140 & 4.9 & SHZ & Bagginidae & Cancris auriculus & -22.5 & 18.9 & Calcareous \\
\hline Pakistan & 140 & 4.9 & SHZ & & G. pyrula, Lenticulina sp., C. auriculus & -22.0 & 1.8 & Calcareous \\
\hline Pakistan & 140 & 4.9 & SHZ & Astrorhizidae & Pelosina sp. & -21.5 & 9.9 & Monothalamid \\
\hline
\end{tabular}




\begin{tabular}{|c|c|c|c|c|c|c|c|c|}
\hline Pakistan & 140 & 4.9 & SHZ & Astrorhizidae & Pelosina sp. & -20.0 & 5.1 & Monothalamid \\
\hline Pakistan & 140 & 4.9 & SHZ & Hormosinidae & Reophax sp. 1 & -23.9 & 8.1 & Textulariid \\
\hline Pakistan & 140 & 4.9 & SHZ & Hormosinidae & Reophax sp. 1 & -22.4 & 19.8 & Textulariid \\
\hline Pakistan & 140 & 4.9 & SHZ & Uvigerinidae & Uvigerina ex. gr. semiornata & -23.7 & 11.4 & Calcareous \\
\hline Pakistan & 140 & 4.9 & SHZ & Uvigerinidae & Uvigerina ex. gr. semiornata & -24.0 & 10.9 & Calcareous \\
\hline Pakistan & 140 & 4.9 & SHZ & Uvigerinidae & Uvigerina ex. gr. semiornata & -23.6 & 11.0 & Calcareous \\
\hline Pakistan & 140 & 4.9 & SHZ & Uvigerinidae & Uvigerina ex. gr. semiornata & -23.3 & 10.5 & Calcareous \\
\hline Pakistan & 140 & 4.9 & SHZ & Uvigerinidae & Uvigerina ex. gr. semiornata & -23.4 & 9.1 & Calcareous \\
\hline Pakistan & 140 & 4.9 & SHZ & Uvigerinidae & Uvigerina ex. gr. semiornata & -20.0 & 5.8 & Calcareous \\
\hline Pakistan & 140 & 4.9 & SHZ & Uvigerinidae & Uvigerina ex. gr. semiornata & -23.2 & 5.8 & Calcareous \\
\hline Pakistan & 140 & 4.9 & SHZ & Uvigerinidae & Uvigerina ex. gr. semiornata & -23.5 & 12.1 & Calcareous \\
\hline Pakistan & 300 & 4.9 & CORE & Buliminidae & Globobulimina cf. G. pyrula & -22.4 & 17.3 & Calcareous \\
\hline Pakistan & 300 & 4.9 & CORE & Buliminidae & Globobulimina cf. G. pyrula & -21.5 & 8.7 & Calcareous \\
\hline Pakistan & 300 & 4.9 & CORE & Astrorhizidae & Pelosina sp. & -19.8 & 12.9 & Monothalamid \\
\hline Pakistan & 300 & 4.9 & CORE & Rhabdamminidae & Bathysiphon sp. & -22.4 & -3.9 & Monothalamid \\
\hline Pakistan & 300 & 4.9 & CORE & Mixture & C. laevigata, C. auriculus, Brizalina & -22.2 & 13.6 & \\
\hline Pakistan & 300 & 4.9 & CORE & Mixture & \& Cribostomoides, Ammodiscus & -22.2 & 13.6 & \\
\hline Pakistan & 300 & 4.9 & CORE & & $\begin{array}{l}\text { Nemogullmia, Saccamminid, Allogromiid, } \\
\text { Casperammina }\end{array}$ & -21.8 & 12.0 & Monothalamid \\
\hline Pakistan & 300 & 4.9 & CORE & & $\begin{array}{l}\text { Saccamminid, Nemogullmia, Allogromiid, } \\
\text { Lagenammina }\end{array}$ & -22.5 & n.d. & Monothalamid \\
\hline Pakistan & 300 & 4.9 & CORE & Hormosinidae & Reophax dentaliniformis & -21.8 & 3.8 & Textulariid \\
\hline Pakistan & 300 & 4.9 & CORE & Hormosinidae & Reophax dentaliniformis & -21.0 & 1.6 & Textulariid \\
\hline Pakistan & 300 & 4.9 & CORE & Hormosinidae & Reophax dentaliniformis & -19.8 & 13.3 & Textulariid \\
\hline Pakistan & 300 & 4.9 & CORE & Hormosinidae & Reophax dentaliniformis & -22.5 & 16.0 & Textulariid \\
\hline Pakistan & 300 & 4.9 & CORE & Hormosinidae & Reophax sp. 2 & -23.5 & 13.1 & Textulariid \\
\hline Pakistan & 300 & 4.9 & CORE & Hormosinidae & Reophax sp. 2 & -22.2 & 12.1 & Textulariid \\
\hline
\end{tabular}




\begin{tabular}{|c|c|c|c|c|c|c|c|c|}
\hline Pakistan & 300 & 4.9 & CORE & Uvigerinidae & Uvigerina ex. gr. semiornata & -22.2 & 7.3 & Calcareous \\
\hline Pakistan & 300 & 4.9 & CORE & Uvigerinidae & Uvigerina ex. gr. semiornata & -22.5 & 13.0 & Calcareous \\
\hline Pakistan & 300 & 4.9 & CORE & Uvigerinidae & Uvigerina ex. gr. semiornata & -19.9 & 12.1 & Calcareous \\
\hline Pakistan & 300 & 4.9 & CORE & Uvigerinidae & Uvigerina ex. gr. semiornata & -18.7 & 12.7 & Calcareous \\
\hline Pakistan & 940 & 7.6 & LTZ & Hormosinidae & R. dentaliniformis, $R$. scorpiurus & -20.6 & 10.0 & Textulariid \\
\hline Pakistan & 940 & 7.6 & LTZ & Chilostomellidae & Chilostomella ovoidea & -21.2 & 11.2 & Calcareous \\
\hline Pakistan & 940 & 7.6 & LTZ & Ammosphaeoidinidae & Cribostomoides & -21.1 & 10.8 & Calcareous \\
\hline Pakistan & 940 & 7.6 & LTZ & Eggerellidae & Dorothia scabra & -25.5 & 27.3 & Textulariid \\
\hline Pakistan & 940 & 7.6 & LTZ & Eggerellidae & Dorothia scabra & -22.5 & n.d. & Textulariid \\
\hline Pakistan & 940 & 7.6 & LTZ & & Bathysiphon, Hyperammina & -22.0 & 10.5 & Monothalamid \\
\hline Pakistan & 940 & 7.6 & LTZ & & Ammodiscus, Cribostomoides, Lenticulina & -20.5 & 11.4 & \\
\hline Pakistan & 940 & 7.6 & LTZ & & \& Karreriella, Globobulimina, Cancris, Cassidulina & -20.5 & 11.4 & \\
\hline Pakistan & 940 & 7.6 & LTZ & Hormosinidae & Reophax aff. bilocularis & -21.3 & 9.0 & Textulariid \\
\hline Pakistan & 940 & 7.6 & LTZ & Hormosinidae & Reophax aff. bilocularis & -21.0 & 9.3 & Textulariid \\
\hline Pakistan & 940 & 7.6 & LTZ & Hormosinidae & Reophax aff. bilocularis & -20.5 & 10.0 & Textulariid \\
\hline Pakistan & 940 & 7.6 & LTZ & Hormosinidae & Reophax aff. bilocularis & -21.0 & 9.0 & Textulariid \\
\hline Pakistan & 940 & 7.6 & LTZ & Hormosinidae & Reophax aff. bilocularis & -21.6 & 9.2 & Textulariid \\
\hline Pakistan & 940 & 7.6 & LTZ & Hormosinidae & Reophax aff. bilocularis & -21.6 & 9.4 & Textulariid \\
\hline Pakistan & 940 & 7.6 & LTZ & Hormosinidae & Reophax aff. bilocularis & -20.5 & 11.2 & Textulariid \\
\hline Pakistan & 940 & 7.6 & LTZ & Hormosinidae & Reophax aff. bilocularis & -20.3 & 10.3 & Textulariid \\
\hline Pakistan & 940 & 7.6 & LTZ & Hormosinidae & Reophax aff. bilocularis & -20.5 & 11.1 & Textulariid \\
\hline Pakistan & 940 & 7.6 & LTZ & Hormosinidae & Reophax aff. bilocularis & -19.4 & 15.2 & Textulariid \\
\hline Pakistan & 1200 & 15.6 & LB & & H. baltica, B. aculeata, C. oolina, C. ovoidea & -22.0 & n.d. & Calcareous \\
\hline Pakistan & 1200 & 15.6 & LB & & \& H. elegans, P. pupoide & -22.0 & n.d. & Calcareous \\
\hline Pakistan & 1200 & 15.6 & LB & & Nodellum, G. aff. pyrula, Melonis, H. baltica & -21.7 & 9.1 & \\
\hline Pakistan & 1200 & 15.6 & LB & & \& Ammodiscus, Cribostomoides, & -21.7 & 9.1 & \\
\hline
\end{tabular}




\begin{tabular}{|c|c|c|c|c|c|c|c|c|}
\hline Pakistan & 1200 & 15.6 & LB & & \& Saccamminids, Bathysiphon, Allogromiid & -21.7 & 9.1 & \\
\hline Pakistan & 1200 & 15.6 & LB & Hormosinidae & Rheophax scorpiurus & -22.3 & 16.0 & Textulariid \\
\hline Pakistan & 1200 & 15.6 & LB & Hormosinidae & R. bilocularis, $R$. scorpiurus, $R$. dentaliniformis & -20.8 & 0.0 & Textulariid \\
\hline Pakistan & 1200 & 15.6 & LB & Hormosinidae & Reophax aff. bilocularis & -20.8 & 10.6 & Textulariid \\
\hline Pakistan & 1200 & 15.6 & LB & Hormosinidae & Reophax aff. bilocularis & -21.6 & 11.7 & Textulariid \\
\hline Pakistan & 1200 & 15.6 & LB & Hormosinidae & Reophax aff. bilocularis & -21.0 & 10.9 & Textulariid \\
\hline Pakistan & 1200 & 15.6 & LB & Hormosinidae & Reophax aff. bilocularis & -21.2 & 11.1 & Textulariid \\
\hline Pakistan & 1200 & 15.6 & LB & Hormosinidae & R. bilocularis, $R$. scorpiurus, $R$. dentaliniformis & -22.0 & 8.6 & Textulariid \\
\hline Pakistan & 1200 & 15.6 & LB & Astrorhizidae & Pelosina sp. & -23.1 & 6.9 & Monothalamid \\
\hline Pakistan & 1850 & 73.6 & BELOW & Komokiidae & Komoki sp. \& Lana sp. & -21.1 & 2.7 & Monothalamid \\
\hline Pakistan & 1850 & 73.6 & BELOW & & Allogromiid, Nodellum, Psammospaerid & -20.6 & 7.2 & Monothalamid \\
\hline Pakistan & 1850 & 73.6 & BELOW & & H. elegans, B. affinis & -19.8 & 10.7 & \\
\hline Pakistan & 1850 & 73.6 & BELOW & & \& Cyclamina sp., Cribostomoides sp. & -19.8 & 10.7 & \\
\hline Pakistan & 1850 & 73.6 & BELOW & Astrorhizidae & Pelosina sp. & -20.7 & 8.7 & Monothalamid \\
\hline Pakistan & 1850 & 73.6 & BELOW & Hormosinidae & R. bilocularis, $R$. scorpiurus, $R$. dentaliniformis & -20.3 & 16.1 & Textulariid \\
\hline Pakistan & 1850 & 73.6 & BELOW & Rhabdamminidae & Rhizammina sp. & -21.1 & 6.7 & Monothalamid \\
\hline Pakistan & 1850 & 73.6 & BELOW & Rhabdamminidae & Rhizammina sp. & -21.4 & 7.8 & Monothalamid \\
\hline Pakistan & 1850 & 73.6 & BELOW & Rhabdamminidae & Rhizammina sp. & -22.1 & 7.2 & Monothalamid \\
\hline Pakistan & 1850 & 73.6 & BELOW & & Nodosium, Bathysiphon, Crithionina & -19.9 & 9.5 & \\
\hline Pakistan & 1850 & 73.6 & BELOW & & \& Cyclamina, Cribostomoides & -19.9 & 9.5 & \\
\hline
\end{tabular}

